\title{
PRÁTICA DA HUMANIZAÇÃO NA VISITA EM UNIDADE DE TERAPIA INTENSIVA
}

\author{
HUMANIZATION PRACTICE DURING INTENSIVE \\ CARE UNIT VISITS
}

\section{PRÁCTICA DE LA HUMANIZACIÓN EN LA VISITA EN UNIDAD DE CUIDADOS INTENSIVOS}

\begin{abstract}
Tamires Alexandre Félix*, Francisco Valdicélio Ferreira**, Eliany Nazaré Oliveira*** Sara Cordeiro Eloia****, Bruna Vieira Gomes****, Suzana Mara Cordeiro Eloia******
\end{abstract}

\begin{abstract}
Autor correspondente: Tamires Alexandre Félix - myrisinha@hotmail.com
*Enfermeira graduada pela Universidade Estadual Vale do Acaraú. Especialista em Gestão dos Serviços de Urgência e Emergência. Especializanda em Terapia Intensiva. Mestranda em Saúde da Família. Sobral, CE, Brasil, **Nutricionista. Especializando em Saúde Pública e Saúde da Família pelo Instituto Superior de Teologia Aplicada. Sobral, CE, Brasil, ***Enfermeira. Doutora em Enfermagem pela Universidade Federal do Ceará (UFC). Docente da Universidade Estadual Vale do Acaraú (UVA). Sobral, CE, Brasil, ${ }^{* * * *}$ Enfermeira. Mestranda em Saúde da Família pela Universidade Federal do Ceará (UFC). Bolsista da Coordenação de Aperfeiçoamento de Pessoal de Nível Superior (CAPES). Sobral, CE, Brasil

*****Enfermeira. Mestranda em Saúde da Família pela Universidade Federal do Ceará (UFC). Bolsista da Fundação Cearense de Apoio ao Desenvolvimento Científico e Tecnológico (FUNCAP). Sobral, CE, Brasil, ${ }^{* * * * * *}$ Acadêmica de Enfermagem pela Universidade Estadual Vale do Acaraú (UVA). Bolsista da Fundação Cearense de Apoio ao Desenvolvimento Científico e Tecnológico (FUNCAP). Sobral, CE, Brasil.
\end{abstract}

\section{Resumo}

A Unidade de Terapia Intensiva (UTI) é a sede terapêutica de maior complexidade em uma unidade hospitalar, onde se centram o máximo de esforços humanos e tecnologias de cuidado visando o pleno reestabelecimento do individuo à sua condição normal ou ao menos a redução do agravo que o conduziu a hospitalização. Vivenciar a internação de um parente em uma UTI requer dos familiares a capacidade de compreender seus próprios sentimentos e elaborar estratégias para o enfrentamento do problema. Este estudo tem o objetivo de conhecer a produção científica acerca da humanização na visita de UTI, reconhecendo como se estabelece a comunicação entre a equipe de enfermagem e os pacientes e seus familiares para desenvolver um relacionamento interpessoal com efeito terapêutico. Trata-se de uma revisão integrativa, método que permite sintetizar o conhecimento produzido e identificar lacunas para fundamentar melhor investigações científicas futuras. Nos resultados foram encontrados oito artigos que abordam a temática, estes concentrados na região sudeste e sul. As categorias temáticas extraídas dos artigos foram concepção e vivência de familiares sobre humanização na visita de UTI, concepção dos profissionais sobre humanização na visita de UTI e estratégias 
de acolhimento. Concluímos que com esta revisão reitera-se a importância de implementar de forma mais efetiva a Política Nacional de Humanização em níveis mais complexos de atenção. Os estudos mostraram realidades adversas, mas embasadas praticamente no mesmo problema estrutural, a falta de comunicação.

Palavras-chave: Humanização, Unidade de Terapia Intensiva, Enfermagem.

\title{
Resumen
}

La Unidad de Cuidados Intensivos (UCI) es la sede terapéutica de mayor complejidad en una unidad hospitalaria, donde se centran el máximo de esfuerzos humanos y tecnologías de cuidado buscando el pleno restablecimiento del individuo a su condición normal o al menos la reducción del agravamiento que lo llevó a la hospitalización. Vivir la internación de un familiar en una UTI requiere de estos la capacidad de comprender sus propios sentimientos y elaborar estrategias para el enfrentamiento del problema. Este estudio tiene por objetivo conocer la producción científica sobre la humanización en la visita de $\mathrm{UCl}$, reconociendo como se establece la comunicación entre el equipo de enfermería y los pacientes y sus familiares para desarrollar un relacionamiento interpersonal con efecto terapéutico. Se trata de una revisión integradora, método que permite sintetizar el conocimiento producido e identificar lagunas para fundamentar mejor, futuras investigaciones científicas. En los resultados fueron encontrados ocho artículos que abordan el tema, estando concentrados en la región sudeste y sur. Las categorías temáticas extraídas de los artículos fueron: concepción y vivencia de familiares sobre humanización en la visita de $\mathrm{UCl}$; concepción de los profesionales sobre humanización en la visita de UCl y estrategias de acogida. Se concluyó que con esta revisión se reitera la importancia de implementar de forma más efectiva la Política Nacional de Humanización a niveles más complejos de atención. Los estudios mostraron realidades adversas, pero basadas prácticamente en el mismo problema estructural, la falta de comunicación.

Palabras clave: Humanización, Unidad de Cuidados Intensivos, Enfermería.

\begin{abstract}
The Intensive Care Unit (ICU) is the highest complexity therapeutic center in a hospital unit, where maximum human effort and care technology are centered seeking to fully reestablish the individual to his/her normal condition or at least reduce the medical problem thatoriginated hospital admission. Experiencing the hospital admission of a family memberin an ICU requires that relatives have the capacity to understand their own feelings and prepare strategies to confront the problem. This study have as objective to find scientific production on humanization in ICU visits, recognizing how communication is established between nursing team and patients and their relatives to develop an interpersonal relationship with therapeutic effect. This study was an integrative review, a method that enables the synthesis of produced knowledge and that identifies loopholes to substantiate better scientific investigations in the future. In results we found eight studies that addressed the theme; these were concentrated in the southeastern and southern regions. The thematic categories extracted from the studies were conception and experience of family members on humanization in $\mathrm{UCl}$ visits, professional conception on humanization in $\mathrm{UCl}$ visits and reception strategies. Conclude that this review reaffirms the importance of implementing the National Humanization Policy in a more effective manner and in more complex care levels. The studies present adverse realities, but they are all virtually basement the same structural problem, the lack of communication.
\end{abstract}

Keywords: Humanization; Intensive Care Unit, Nursing. 


\section{INTRODUÇÃO}

A Unidade de Terapia Intensiva (UTI) é a sede terapêutica de maior complexidade em uma unidade hospitalar. Nela, centram-se o máximo de esforços humanos e tecnologias de cuidado visando o pleno reestabelecimento do individuo à sua condição normal ou ao menos a redução do agravo que o conduziu a hospitalização.

Vivenciar a internação de um parente em uma UTI requer dos familiares a capacidade de compreender seus próprios sentimentos e elaborar estratégias para o enfrentamento do problema. É um momento de extrema vulnerabilidade em que o paciente tem seu contato reduzido com seus parentes, permanecendo integralmente sob os cuidados de uma equipe intensivista.

A necessidade de internar um familiar em uma UTI tanto pode provocar sentimentos de esperança, alívio, conforto, como de temor e insegurança, dentre outros. ${ }^{(1)}$

Para os pacientes, o único momento proporcionado ao contato com familiares é o intervalo limitado destinado à visita. Neste momento, os familiares "despejam" seus anseios e emoções, suas dúvidas com relação à evolução da doença, suas preocupações e reflexos da quebra de papéis sociais proporcionada pelo internamento. A maioria dos pacientes, em estado grave, não responde satisfatoriamente aos estímulos o que direciona os familiares aos profissionais de saúde.

As informações oferecidas pelos profissionais no momento da visita, geralmente médicos e enfermeiros, devem ser clarificadoras e prestadas de forma empática, o que requer dos profissionais uma boa relação de comunicação e entendimento da relação terapêutica produzida através do contato entre família e paciente. Raramente os parentes contam com serviços de apoio social e psicologia para vivenciarem de maneira menos sofredora a internação do ente querido.

Concorda-se com os autores ${ }^{(2: 531)}$ que "é por meio da comunicação estabelecida com os pacientes e seus familiares que podemos compreendê-los em seu todo. Só assim poderemos identificar seus problemas e ajudá-los". Assim, fica explícita a necessidade da interação humana na prestação de cuidado, motivo pelo qual se considera a comunicação um relevante fator de humanização na UTI.

A comunicação é a base fundamental nessa relação enfermeiro-usuário estabelecida na prática assistencial. Nela estão presentes formas de comunicação e expressão diferenciadas, aspectos culturais, vivências, conhecimento empírico e científico, crenças e valores, tendo subjacente a si, o próprio modelo conceitual de enfermagem em que se enraíza. (3)

Como exemplo, cita-se um estudo(4) que justificou esta abordagem à luz da Teoria das Relações Interpessoais de Travelbee. Ajudar a família a encontrar sentido e entender que representações psico-socio-espirituais tem a doença é papel do enfermeiro. No estudo apresentam-se estratégias para estabelecer um bom relacionamento interpessoal entre enfermeiro-paciente-família na visita de UTI, descrevendo técnicas de comunicação que mudem o estereótipo do enfermeiro intensivista como um profissional puramente técnico, que não se comunica e não leva em consideração a estrutura familiar no processo de cuidar.

Percebe-se, portanto, que a humanização em UTI perpassa por um cuidado livre de juízos de valor e alcança um elo com a família a ponto de estabelecer uma relação terapêutica que favoreça a reabilitação do doente. Para tal, o momento da visita de parentes na UTI deve ser bem construído e bem vivido, o que poucas vezes acontece.

Destacando, neste contexto, o profissional de enfermagem que possui papel fundamental na equipe intensivista, é imprescindível que adquira competências necessárias à sua prática profissional, traduzidas pela associação de conhecimentos, habilidades e atitudes, e que busque renovar-se continuamente, desenvolvendo um olhar crítico e investigador. ${ }^{(5)}$ Neste sentido, tem-se como pressuposto que se eles tiverem a oportunidade de aprofundar o conhecimento sobre o acolhimento em UTI, suas práticas de cuidado estarão alicerçadas no respeito 
e na subjetividade dos sujeitos envolvidos e sentirão que o cuidado oferecido é integral e eficaz. ${ }^{(6)}$

Em concordância, outros estudiosos ${ }^{(7)}$ afirmam que a enfermagem é que encadeia a construção do cuidado efetivo em decorrência de sua inserção nos processos de trabalho e seu potencial articulador, valorizando o profissional que desenvolve esta habilidade e consegue planejar os cuidados ao paciente, atender às suas necessidades e incluir a família como parte deste processo.

A equipe precisa considerar as necessidades da família diante de situações estressantes e estabelecer um plano de cuidados que seja construído e continuamente validado, avaliado e reavaliado. Desta forma, a interação da equipe de enfermagem com os familiares e o paciente será estabelecida através do diálogo e da busca dos significados que as experiências de doença geram em cada pessoa.

Neste contexto, este estudo objetiva conhecer a produção científica acerca da humanização na visita de UTI, reconhecendo como se estabelece a comunicação da equipe com os pacientes e seus familiares para desenvolver o relacionamento interpessoal com efeito terapêutico.

\section{MÉTODOS}

O presente estudo trata-se de uma revisão integrativa. Este método permite sintetizar o conhecimento produzido e identificar lacunas para fundamentar melhor investigações científicas futuras. A revisão integrativa permite visualizar os achados $\mathrm{e}$ reflete de maneira geral o conhecimento produzido por estudos independentes.

Esta produção seguiu a descrição das etapas percorridas para a elaboração de uma revisão integrativa, a saber: elaboração da pergunta norteadora; busca ou amostragem na literatura; coleta de dados; análise crítica dos estudos incluídos; discussão dos resultados e apresentação da revisão integrativa. ${ }^{(8)}$

As questões que nortearam este estudo foram: Como se estabelece a visita em uma UTI? De que forma a humanização tem-se incorporado à interação dos familiares com o paciente e os profissionais de saúde?

Empreendeu-se a busca dos artigos no mês de maio de 2013, ao processar as palavras-chaves humanização, unidade de terapia intensiva e visita, acessando as bases de dados Scientific Electronic Library Online (SCIELO), Literatura Latino-Americana e do Caribe em Ciências da Saúde (LILACS) e Banco de Dados em Enfermagem (BDENF). É importante ressaltar que tais bases de dados são reconhecidas pela ampla discussão científica.

Os critérios de inclusão para a seleção dos artigos foram: estarem disponíveis na íntegra, apresentação na língua portuguesa, publicados nas bases de dados referidas, no período de 2008 a maio de 2013, e que abordassem a temática proposta. Excluíram-se aqueles artigos repetidos em mais de uma base de dados e que não se relacionaram aos objetivos. Desta forma, obteve-se uma amostra de 8 artigos.

Em seguida, para reunir e sintetizar as informações-chave, elaborou-se um formulário com os itens de análise: ano de publicação, periódico, região da pesquisa,formação profissional dos autores,título, metodologia empregada e principais resultados de forma a organizar os dados das produções incluídas nesta revisão, facilitando a checagem e reduzindo erros de análise.

Procedendo a leitura do título e resumo de cada artigo científico, pôde-se verificar conformidade com as questões norteadoras da presente investigação. Posteriormente, realizou-se a leitura na íntegra de cada estudo para categorização dos mesmos. A categorização baseou-se na extração das informações principais apresentadas nas pesquisas agrupando por similaridade de conteúdo os estudos com temas semelhantes. ${ }^{(9)}$

\section{RESULTADOS}

Do total de 8 artigos, constatou-se que 3 (37,5\%) foram publicados no ano de 2009, $2(25,0 \%)$ no 
ano de 2011 e 1(12,5\%) artigo nos anos de 2008, 2010 e 2012. Nenhum artigo foi publicado no ano corrente, até maio de 2013.

Em relação à região em que se concentram as produções desta revisão, apresenta-se a região Sul como cenário de $5(62,5 \%)$ estudos, seguida da região Sudeste com 3 (37,5\%). Em contrapartida, não se identificaram produções nas demais regiões.

Ao analisar o periódico de publicação, 3 (37,5\%) estudos foram publicados pela Revista de Enfermagem da UERJ, 2 (25,0\%) pela Ciência, Cuidado e Saúde, 1 (12,5\%) pelos Arquivos de Ciências da Saúde da UNIPAR, assim como, no Jornal Brasilei- ro de Psiquiatria e na Escola Anna Nery Revista de Enfermagem.

Quanto à metodologia empregada nestes estudos, 7 (87,5\%) dispôs-se da abordagem qualitativa, como também 7 (87,5\%) das publicações foram de autoria exclusiva de enfermeiros.

Pela análise e interpretação dos resultados, emergiram três categorias temáticas: Concepção e Vivência de familiares sobre humanização na visita de UTI, Concepção dos profissionais sobre humanização na visita de UTI e Estratégias de acolhimento. A Tabela 1 apresenta as categorias temáticas e os correspondentes títulos dos estudos.

Tabela 1- Categorias temáticas e títulos dos estudos. Sobral, Ceará, Brasil, 2013.

\begin{tabular}{|l|l|}
\hline \multicolumn{1}{|c|}{ CATEGoRIAS TEMÁtICAS } & \multicolumn{1}{c|}{ TítULOS DOS ESTUDos } \\
\hline $\begin{array}{l}\text { Concepção e vivência de } \\
\text { familiares sobre humanização na } \\
\text { visita de UTI }\end{array}$ & $\begin{array}{l}\text { Visita em unidades de terapia intensiva: concepção dos familiares quanto } \\
\text { à humanização do atendimento; } \\
\text { Fatores estressantes para familiares de pacientes criticamente enfermos } \\
\text { de uma unidade de terapia intensiva; } \\
\text { Comunicação entre profissional de saúde e familiares de pacientes em } \\
\text { terapia intensiva; } \\
\text { Implantação de tecnologias de cuidado em unidade de terapia intensiva } \\
\text { aos usuários e seus familiares. }\end{array}$ \\
\hline $\begin{array}{l}\text { Concepção dos profissionais } \\
\text { UTI }\end{array}$ & $\begin{array}{l}\text { Percepção da equipe de saúde sobre a família na UTI neonatal: resistência } \\
\text { aos novos saberes; } \\
\text { A visita de familiares em unidades intensivas na ótica da equipe de } \\
\text { enfermagem; } \\
\text { Discursos de enfermeiros sobre humanização na unidade de terapia } \\
\text { intensiva. }\end{array}$ \\
\hline Estratégias de Acolhimento & $\begin{array}{l}\text { Estratégias para o acolhimento dos familiares dos pacientes na unidade } \\
\text { de terapia intensiva. }\end{array}$ \\
\hline
\end{tabular}

Fonte: , LILACS E BDENF

\section{DISCUSSÃO}

Considerando a publicação da Politica Nacional de Humanização(10) como o marco incentivador às práticas humanísticas do âmbito do SUS, entende-se que a partir deste ponto e de forma crescente devem-se apresentar pesquisas voltadas para o cuidado humanizado na área da saúde de forma a implementá-lo e aprimorá-lo. No entanto, é necessária maior produção científica neste contexto tendo em vista a quantidade mínima de artigos selecionados neste estudo.

Com relação à região em que se concentram as produções esta revisão apresenta a região Sul como cenário de 5 das 8 pesquisas. Em contrapartida Norte, Nordeste e Centro-Oeste frente aos critérios deste estudo tem produção escassa ou nula com relação à humanização na visita de UTI. 
Entre 2005 e 2013, o número de leitos de UTI no Brasil cresceu $36 \%$. Em estudo realizado por Almeida ${ }^{(11)}$ sobre a oferta de leitos de UTI no Brasil a região Norte apresentou o melhor índice nacional de crescimento enquanto a região sudeste apresentou o pior índice. Desta forma, as regiões que menos produzem acerca da humanização na visita de UTI tendem a ser as que mais dispõem do serviço. Nota-se também que nenhuma pesquisa inclusa envolveu o âmbito nacional como cenário de estudo.

Identificou-se, também, que a maioria dos estudos resultava de pesquisas de profissionais enfermeiros. Tal fato reflete a preocupação e interação do profissional com a humanização na terapia intensiva apesar de esta ser uma ação que compete à equipe interdisciplinar. Podemos, a partir deste resultado, questionar: Por que as demais categorias não investigam acerca da temática? Pode o enfermeiro fazer humanização de forma isolada?

A seguir, apresentam-se as categorias temáticas resultantes da análise e interpretação dos resultados destes artigos.

\section{CONCEPÇÃO E VIVÊNCIA DE FAMILIARES SOBRE HUMANIZAÇÃO NA VISITA DE UTI}

Abordando familiares sobre a percepção da vivência do internamento e da abordagem da equipe intensivista, a maioria dos estudos aponta para certa insatisfação relacionada à falta de empatia por parte dos profissionais, pouca valorização da experiência e pouco apoio da instituição hospitalar no processo.

Portanto, é preciso que haja interação entre quem cuida e quem é cuidado adotando uma prática em que paciente, familiares e profissionais participem juntos do processo terapêutico, fortalecendo uma postura ética, humana, reflexiva e o cuidado técno-científico.

Um estudo realizado em um hospital de ensino do noroeste paulista abordou 41 familiares sobre a humanização no horário da visita de UTI. Entre os resultados, destaca-se que $43,9 \%$ da amostra afirmou que não houve esclarecimentos sobre a unidade, seu funcionamento, normas e finalidade; $70,7 \%$ dos familiares afirmaram nem sequer conhecer o enfermeiro da UTI; 65,8\% aprovam a duração da visita por ol hora. Os autores citaram que a maioria dos familiares associa ao médico o papel de informar sobre o estado do paciente, o que gera discussão acerca da orientação cultural dos familiares e do papel desempenhado pelo enfermeiro, demonstrando que a assistência de enfermagem humanizada às famílias ainda possui falhas e as orientações feitas por este profissional ainda são escassas $^{(12)}$.

Vê-se como prejudicial o não esclarecimento sobre a rotina na UTI, visto que uma explicação mais detalhada para os familiares sobre os motivos que levam a essa rotina poderia reduzir o estresse percebido. ${ }^{(13)}$

Neste contexto, apresentam-se sugestões dos familiares para melhorar a humanização no atendimento, tais como: adicionar mais um horário de visita e permitir entrada extra de parentes em casos de extrema gravidade, bem como de pessoas que possam oferecer conforto espiritual. Ansiedade e angústia foram os sentimentos mais citados. ${ }^{(12)}$ Este perfil de opiniões e percepções também surge em outros estudos. ${ }^{(13-15)}$

A literatura tem apontado altos níveis de ansiedade e sintomas depressivos entre membros de familiares de pacientes internados em UTI; diante disso o cuidado tem-se estendido aos familiares no intuito de conhecer e reduzir a ocorrência de fatores estressantes.

Em um dos estudos ${ }^{(13)}$ aplicou-se uma escala com 25 fatores estressantes a 53 familiares de pacientes internados numa UTI. Fatores relacionados aos pacientes obtiveram as maiores somatórias, como ver o paciente contido, sem roupa e o tempo de internação. Em seguida surgiram os fatores relacionados à visita, ao ambiente e por último à equipe. $O$ atraso no horário de visitas, o excesso de aparelhos ao redor do paciente e o contato reduzido com a equipe estão entre os itens mais citados pelos familiares. 
Pelos resultados desta pesquisa, foi possível perceber que apesar das limitações impostas por profissionais, os familiares se preocupam mais com o paciente e os fatores que interagem diretamente com ele, do que propriamente com o desempenho técnico e humano da equipe cuidadora. ${ }^{\left({ }^{(3)}\right.}$

Considerando a pesquisa de Marques, Silva e Maia ${ }^{(14)}$ que abordou 22 famílias após a alta do paciente, é possível ver reflexos de práticas não humanizadas. A maioria das famílias (54,5\%) indicou que reconhecia a UTI como lugar que trata de doenças graves e que se sentia satisfeita com os profissionais, inclusive enfermeiros, seu cuidado humanizado e assistência prestada. No entanto, os mesmos familiares se contradizem ao relatarem a necessidade de mais atenção, apoio e esclarecimentos. Estes autores justificam esses dados ao elevado grau de estresse emocional enunciado pelos fatores já apresentados no estudo anterior ${ }^{(13)}$. Algumas famílias relataram gratidão e satisfação ao atendimento prestado além da necessidade de manter vínculo após a alta.

Outro estudo(15) objetivou abordar concepções e vivências de familiares, colhendo opiniões de 15 sujeitos acerca da tecnologia de cuidado, distribuindo um manual explicativo, acolhendo-os e estabelecendo interações. A partir das experiências vivenciadas na UTI e dos relatos dos participantes, considerou-se que estas interações foram favoráveis ao cuidado.

Neste contexto, surge a dimensão ética do uso de tecnologias de cuidado com a aplicação do saber da enfermagem, ao direcionar o atendimento às necessidades dos clientes, usuários e familiares, com ações menos verticalizadas e em permanente processo de reflexão, interpretação e construção. ${ }^{(15)}$

A humanização na visita de UTI requer, sob visão dos familiares, um processo de avaliação e reavaliação constante. As necessidades julgadas mais importantes pela família se referem ao interesse dos profissionais de UTI pelo paciente; estar seguro de que o melhor tratamento possível está sendo oferecido; sentir que há esperança de melhora; ter perguntas respondidas com franqueza. ${ }^{(16)}$ Portan- to, cabe à equipe multiprofissional que assiste aos pacientes críticos abordar cada grupo familiar de forma singular, mas especificamente no momento da visita, considerando-a como espaço para práticas de humanização.

\section{CONCEPÇÃO DOS PROFISSIONAIS SOBRE HUMANIZAÇÃO NA VISITA DE UTI}

Como já citado anteriormente, a maioria dos artigos é de autoria exclusiva de profissionais enfermeiros, o que gerou uma tendência na discussão deste tópico em apresentar a concepção da enfermagem. Dos 3 artigos que abordaram esta temática, somente um incluiu a categoria médica em sua amostra.

Em um estudo, ${ }^{(17)}$ os profissionais de enfermagem em terapia intensiva foram abordados acerca de 3 temas: o distanciamento entre a equipe e familiares nas unidades intensivas, o desconhecimento dos familiares sobre a condição do paciente e o ambiente das unidades intensivas, e atitudes adotadas pela equipe na visita dos familiares. Os resultados demonstraram que o envolvimento da equipe de enfermagem com a família no período da visita, de modo geral, ainda não é percebido como uma prática de cuidado.

Conforme afirmam Casanova e Lopes, ${ }^{(18)}$ o horário da visita não é utilizado pelos integrantes da equipe de enfermagem como uma oportunidade de comunicação e diálogo com o paciente e o seu familiar, sendo necessário o desenvolvimento de estratégias de escuta para reverter esta situação.

De maneira geral, muitos fatores foram apresentados pelos profissionais como elementos de distanciamento entre a equipe e a família, a saber: medo de prestar esclarecimentos de forma equivocada ou que possam preocupar ainda mais os familiares, barreiras culturais, excesso de responsabilidades técnicas, ausência de atividades de sensibilização sobre a humanização na UTI, bem como momentos de escuta de familiares, atribuição da tarefa de informar e interagir especificamente ao profissional médico. ${ }^{(17)}$ 
Quanto ao desconhecimento dos familiares em relação ao processo de reabilitação de seu familiar é possível notar na prática a dificuldade dos parentes de compreender a condição clínica do paciente e até de reconhecê-lo fisicamente. Cabe, portanto, à equipe multiprofissional corresponder às expectativas dos familiares de maneira holística. O profissional que recebe a família necessita ter conhecimento e sensibilidade para oferecer explicações claras e acessíveis sobre o estado de saúde, tratamento e equipamentos usados pelo paciente. Assim, à medida que os familiares vêm para outras visitas, as informações poderão ser somente complementadas. ${ }^{(17)}$

A falta de informação prestada aos familiares, inclusive sobre a UTI, demonstra que a Política de Humanização ainda não é totalmente efetivada em níveis mais complexos de atenção.

É direito dos familiares conhecer o ambiente da UTI, seu funcionamento e estrutura, bem como os profissionais que irão atuar diretamente sobre o paciente $^{(17)}$ Quando isto não acontece, os familiares geralmente se defrontam com um cenário desconhecido durante a visita, aumentando o nível de estresse e insatisfação.

Tratando das atitudes adotadas pela equipe para favorecer a humanização no horário de visita, a maioria dos discursos apresentou a preparação de um ambiente propício para receber a família com o mínimo de ruídos possíveis, sem conversas informais, limpo e organizado. A reflexão proporcionada por estudos como este ajuda a sensibilizar acerca do tema e favorece o desenvolvimento de novas concepções que incluam ações diferenciadas no acolhimento em UTI. ${ }^{(17)}$

Há elementos da prática dos enfermeiros intensivistas que dificultam a implementação da humanização da assistência. Observou-se que, por vezes, a assistência é mais voltada para o paciente/ grupo familiar de maior afinidade com a equipe ou de maior gravidade e interesse clínico. Tratando a família enquanto sujeito de cuidado, é fato que a abordagem não deve ser sintética, que presta e coleta informações em tom de entrevista, mas sim a que constrói sentidos com informações pessoais e sociais ligadas ao ambiente externo. ${ }^{(17,19)}$

O medo dos profissionais de envolver-se pessoalmente nos casos, a falta de interdisciplinaridade nas ações e de um espaço próprio para a acolhida de familiares foram citados como fatores limitantes para a humanização. Vê-se, portanto, que a humanização envolve também o cuidado à equipe de enfermagem. É necessário criar meios para que o profissional enfrente as tensões e sentimentos, não somente os dele, mas os do usuário que ele cuida e do familiar. ${ }^{(19)}$

Portanto, é de extrema relevância discutir acerca do assunto buscando de forma prática colocar a comunicação como item prescrito para cada paciente e seu grupo familiar. A falta de comunicação frequentemente se materializa num cuidado técnico e desumano reclamado pelos familiares.

Conforme Marques, Silva e Maia ${ }^{(14)}$ a comunicação é estratégia básica de comunicação e instrumento único no cuidar de enfermagem em terapia intensiva otimizando o exercício de autonomia e produzindo interação terapêutica entre quem cuida e quem é cuidado. Os autores ainda sugerem que é preciso criar estratégias e adaptar-se a cada grupo familiar para conduzir o processo de reabilitação.

Enfermeiros, técnicos de enfermagem e médicos que trabalham numa UTI neonatal perceberam a restrição de familiares como algo positivo, relacionando ao reconhecimento de uma determinada relação de poder da equipe sobre a família, o ato de informar a família como uma atribuição a mais na rotina já saturada, o tempo de visita sendo um fator que reduz as intervenções técnicas e a presença de parentes atuando como verdadeiros "fiscais". (20) Assim, tratando desta humanização, considera-se que produzir ações e reações cristalizadas não condiz com as determinações de uma assistência adequada.

\section{ESTRATÉGIAS DE ACOLHIMENTO}

Diante do exposto é preciso apresentar meios para que profissionais com intenções transformadoras 
possam iniciar o processo de mudança e humanização em seu ambiente de trabalho. Tratando especificamente do ambiente de UTI e do horário da visita é possível traçar estratégias iniciais que irão sensibilizar a equipe e incluir paulatinamente a família no projeto terapêutico, despertando os profissionais a atitudes mais humanas.

É sabido que não se pode elencar estratégias e aplicá-las somente. Vale considerar a necessidade de adaptar-se a cada realidade, ao contexto sociocultural em que estão inseridos os atores do processo e a estrutura do hospital.

Foram identificadas estratégias de acolhimento implementadas por enfermeiros de uma UTI no Sul do Brasil bastantes válidas para aplicação em demais realidades, tais como: determinar um espaço para a acolhida dos familiares permitindo estabelecer vínculo de confiança sem interpor a posição de profissional; explicar aos familiares de forma acessível a rotina de UTI, seu ambiente e estrutura, bem como os cuidados necessários na hora da visita; designar de forma alternada profissionais médicos, enfermeiros, psicólogos e assistentes sociais para informar e acompanhar o familiar no horário de visita; manter vínculo por telefone em horários distintos; prestar informações claras e valorizar a experiência em tom de partilha e compreensão; colher informações demonstrando interesse pelo paciente; permitir espaço reservado para contato pessoal entre familiares e pacientes; manter um ambiente harmônico para o horário de visita; estabelecer um núcleo de humanização interno que considere casos e determine ações para a equipe; realizar ações de sensibilização de forma contínua; e acompanhar o familiar até a saída. ${ }^{(6)}$

Outras estratégias devem ser desenvolvidas frutos da experiência em cada espaço de terapia intensiva. Devem surgir meios criativos de lidar com adversidades e limitações para humanização em ambiente de UTI e que estes meios sejam divulgados de forma a disseminar conhecimento sobre a temática e favorecer a prática em questão, bem como na formação acadêmica.

\section{CONCLUSÃO}

Com esta revisão, reitera-se a importância de implementar de forma mais efetiva a Politica Nacional de Humanização em níveis mais complexos de atenção. Os estudos mostraram realidades adversas, mas embasadas praticamente no mesmo problema estrutural, a falta de comunicação.

A comunicação como ferramenta terapêutica é o meio que possibilita inverter uma realidade de um cuidado tecnicista para abordagens subjetivas que incluam a família como sujeita de cuidado e coparticipante na assistência ao paciente.

A inserção do profissional enfermeiro como o que mais publica acerca da temática gera questionamento acerca do trabalho interdisciplinar no ambiente de UTI e da dificuldade de desconstruir pensamentos e racionalidades hegemônicos sobre a participação da família na assistência.

Notável a falta de qualificação dos profissionais para efetivar a humanização em ambientes hospitalares, especificamente na terapia intensiva, evidenciado pelo vínculo fraco estabelecido com os grupos familiares demonstrado nos estudos abordados. Vê-se que no campo conceitual a enfermagem tem crescido, mas no campo da prática a visita ainda não se estabeleceu como espaço terapêutico e de cuidado de enfermagem.

A comunicação ainda tem sido restrita, demandando abordagens mais amplas que contextualizem melhor as discussões e relatem experiências e ações interdisciplinares. É preciso reorganizar o cenário de trabalho considerando o horário de visita e estabelecendo ações voltadas para o sujeito e não para a doença.

Este estudo possui limitações quanto a sua metodologia. Primeiro, refere-se ao uso de somente 3 bases de dados não considera outros idiomas, além do português. Entretanto, possibilitou uma análise da produção científica sobre a humanização na visita do familiar em UTI e propõe ao aprofundamento da temática em estudos posteriores, como também, a divulgação de estratégias na prática cotidiana. 


\section{REFERÊNCIAS}

1. Silveira RS, Lunardi VL, Lunardi Filho WD, Oliveira AMN. Uma tentativa de humanizar a relação da equipe de enfermagem com a família de pacientes internados na UTI. Texto e Contexto Enferm [Internet]. 2005 [cited 2013 june 10];14(Esp.):125-30. Available from: http:// www.scielo.br/pdf/tce/v14nspe/a15v14nspe.pdf.

2. Schneider CC, Bielemann VLM, Sousa AS, Quadros LCM, Kantorski LP. Comunicação na Unidade de Tratamento Intensivo, importância e limites - visão da enfermagem e familiares. Cienc. Cuid. Saude [Internet]. 2009 Oct-Dec [cited 2013 june 10]; 8(4):531-9. Available from: http://periodicos.uem.br/ojs/index.php/ CiencCuidSaude/article/view/9667/5384.

3. Haddad JGV, Machado EP, Neves-Amado J, Zoboli ELCP. A comunicação terapêutica na relação enfermeiro-usuário da atenção básica: um instrumento para a promoção da saúde e cidadania. Mundo Saúde [Internet]. 2011 [cited 2013 june 10]; 35(2):145-55. Available from: http://www.saocamilo-sp.br/pdf/mundo_ saude/84/145-155.pdf.

4. Waidman MAP, Elsen I, Marcon SS.

Possibilidades e limites da teoria de Joyce Travelbee para a construção de uma metodologia de cuidado à família. REE [Internet]. 2006 [cited 2013 june 11]; 8(2):28291. Available from: http://www.fen.ufg.br/revista/ revista8_2/v8n2al3.htm.

5. Barreto VPM, Tonini T, Aguiar BGC. Abordagem das competências necessárias ao enfermeiro intensivista: estudo de revisão de literatura. Rev Enferm UFPE on line [Internet]. 2009 July-Sept [cited 2013 june 11]; 3(2):671-7. Available from: http://www.revista.ufpe.br/revistaenfermagem/ index.php/revista/article/view/179/pdf_920.

6. Maestri E, Nascimento ERP, Bertoncello KCG, Martins JJ. Avaliação das estratégias de acolhimento na unidade de terapia intensiva. Rev Esc Enferm USP [Internet]. 2012 Feb [cited 2013 june 11]; 46(1):75-81. Available from: http:// www.scielo.br/pdf/reeusp/v46nl/v46nla10.pdf.

7. Oliveira EM, Spiri WC. O Significado do Processo de trabalho Cuidar para o enfermeiro da UTI. Cienc. Cuid. Saude [Internet]. 2011 JulSep [cited 2013 june 16];1O(3):482-9. Available from: http://periodicos.uem.br/ojs/index.php/ CiencCuidSaude/article/viewFile/11015/pdf.

8. Souza MT, Silva MD, Carvalho R. Revisão integrativa: o que é e como fazer. Einstein [Internet]. 2010 [cited 2013 june 16];8(1 Pt 1):1O2-6. Available from: http://apps.einstein.br/ revista/arquivos/PDF/1134-Einsteinv8n1_p1O2106_port.pdf.

9. Bardin L. Análise de conteúdo. Lisboa: Edições 70; 1977.

10. Brasil. Ministério da Saúde (MS). SecretariaExecutiva, Núcleo Técnico da Política Nacional de Humanização. HumanizaSUS: Política Nacional de Humanização: a humanização como eixo norteador das práticas de atenção e gestão em todas as instâncias do SUS. Brasília: MS; 2004.

11. Almeida ANPM. Análise da oferta de leitos de UTI no Brasil [monografia]. Porto Alegre: Universidade Federal do Rio Grande do Sul. Curso de Especialização em Políticas Públicas, 2006.

12. Beccaria LM, Ribeiro R, Souza GL, Scarpetti N, Contrin LM, Pereira RAM, et al. Visita em Unidades de Terapia Intensiva: concepção dos familiares quanto à humanização do atendimento. Arq. Cienc. Saude [Internet]. 2008 Apr-June [cited 2013 june 20];15(2):659. Available from: http://www.cienciasdasaude. famerp.br/racs_ol/vol-15-2/id\%2O263.pdf.

13. Costa JB, Felicetti CR, Costa CRLM, Miglioranza DC, Osaku EF, Versa GLGS, et al. Fatores estressantes para familiares de pacientes criticamente enfermos de uma unidade de terapia intensiva. J Bras Psiquiatr [Internet]. 2010 [cited 2013 june 2O];59(3):1829. Available from: http://www.scielo.br/pdf/ jbpsiq/v59n3/aO3v59n3.pdf.

14. Marques RC, Silva MJP, Maia FOM. Comunicação entre profissional de saúde e familiares de pacientes em terapia intensiva. Rev Enferm UERJ [Internet]. 2009 Jan-Mar [cited 2013 june 20];17(1):91-5. Available from: http://www.facenf.uerj.br/v17n1/v17nla17.pdf.

15. Zacarias CC, Silveira RS, Lunardi VL, Cappellaro J, Pinto EMS, Lunardi Filho WD. 
Implantação de tecnologias de cuidado em Unidade de Terapia Intensiva aos usuários e seus familiares. Cienc. Cuid. Saude [Internet]. 2009 Apr-June [cited 2013 june 23];8(2):161-

8. Available from: http://periodicos.uem. br/ojs/index.php/CiencCuidSaude/article/ viewFile/8194/4922.

16. Morgon FH, Guirardello EB. Validação da escala de razão das necessidades de familiares em Unidade de terapia Intensiva. Rev Lat Am Enfermagem [Internet]. 2004 Mar-Apr [cited 2013 june 23]; 12(2):198-203. Available from: http://www.scielo.br/pdf/rlae/v12n2/ v12n2aO8.pdf.

17. Predebon GR, Beuter M, Flores RG, GirardonPerlini NMO, Brondani CM, Santos NO. A visita de familiares em unidades intensivas na ótica da equipe de enfermagem. Cienc. Cuid. Saude [Internet]. 2011 [cited 2013 june 23];1O(4):70512. Available from: http://periodicos.vem.br/ojs/
index.php/CiencCuidSaude/article/view/18314/ pdf.

18. Casanova EG, Lopes GT. Comunicação da equipe de enfermagem com a família do paciente. Rev Bras Enferm [Internet]. 2009 Nov-Dec [cited 2013 june 23];62(6):831-6. Available from: http://www.scielo.br/pdf/reben/ v62n6/aO5v62n6.pdf.

19. Silva FD, Chernicharo IM, Silva RC, Ferreira MA. Discursos de enfermeiros sobre humanização na Unidade de Terapia Intensiva. Esc. Anna Nery. 2O12 Oct-Dec;16(4):719-27.

2O. Costa R, Padilha MI. Percepção da equipe de saúde sobre a família na UTI Neonatal: resistência aos novos saberes. Rev Enferm UERJ [Internet]. 2011 Apr-June [cited 2013 june 27];19(2):231-5. Available from: http://www. facenf.uerj.br/v19n2/v19n2a1O.pdf. 